\title{
Class VI Unconventional Myosin is Required for Spermatogenesis in Drosophila
}

\author{
Jennifer L. Hicks, ${ }^{*}$ Wu-Min Deng, ${ }^{\dagger \ddagger}$ Aaron D. Rogat, ${ }^{*}$ Kathryn G. Miller, ${ }^{* \S}$ \\ and Mary Bownes ${ }^{+}$
}

\author{
*Department of Biology, Washington University, St. Louis, Missouri 63130; and 'University of \\ Edinburgh, Institute of Cell and Molecular Biology, Edinburgh EH9 3JR, United Kingdom
}

Submitted May 20, 1999; Accepted September 24, 1999

Monitoring Editor: Paul T. Matsudaira

\begin{abstract}
We have identified partial loss of function mutations in class VI unconventional myosin, 95F myosin, which results in male sterility. During spermatogenesis the germ line precursor cells undergo mitosis and meiosis to form a bundle of 64 spermatids. The spermatids remain interconnected by cytoplasmic bridges until individualization. The process of individualization involves the formation of a complex of cytoskeletal proteins and membrane, the individualization complex (IC), around the spermatid nuclei. This complex traverses the length of each spermatid resolving the shared membrane into a single membrane enclosing each spermatid. We have determined that $95 \mathrm{~F}$ myosin is a component of the IC whose function is essential for individualization. In wild-type testes, $95 \mathrm{~F}$ myosin localizes to the leading edge of the IC. Two independent mutations in 95F myosin reduce the amount of 95F myosin in only a subset of tissues, including the testes. This reduction of $95 \mathrm{~F}$ myosin causes male sterility as a result of defects in spermatid individualization. Germ line transformation with the 95F myosin heavy chain cDNA rescues the male sterility phenotype. IC movement is aberrant in these $95 \mathrm{~F}$ myosin mutants, indicating a critical role for 95F myosin in IC movement. This report is the first identification of a component of the IC other than actin. We propose that $95 \mathrm{~F}$ myosin is a motor that participates in membrane reorganization during individualization.
\end{abstract}

\section{INTRODUCTION}

In Drosophila mature sperm arise from stem cells that reside at the tip of each testis. These stem cells undergo four synchronous rounds of mitosis without cellular division to yield 16 spermatocytes encased by two cyst cells of somatic origin. The 16 spermatocytes undergo meiosis in the midregion of the testis, resulting in 64 spermatids connected by a network of cytoplasmic bridges. Still in a syncytium, the spermatids elongate the length of the testis, reaching $\sim 2 \mathrm{~mm}$ in size (see Figure 5A for schematic; for reviews, see Lindsley and Tokuyasu, 1980; Fuller, 1993).

To be competent for fertilization each spermatid must be enclosed in its own membrane by a process called individualization (see Figure 1 for a schematic of individualization). Individualization proceeds from the head region of each spermatid to the tail (Tokuyasu et al., 1972). The cystic bulge, a spindle-shaped enlargement of the cyst, is the region in which individualization is occurring. Within the bulge the individualized portion is devoid of cytoplasmic organelles

\footnotetext{
‡ Present address: J579, HSB, Department of Biochemistry, Box 357350, University of Washington, Seattle, WA 98195.

§ Corresponding author. E-mail address: miller@biology.wustl.edu.
}

(except the major and minor mitochondrial derivatives and the axoneme) and contains structures termed investment cones. A single investment cone, filled with fine fibrils $\sim 60$ $\AA$ in diameter, surrounds each spermatid. Golgi reside at the individualization boundary, and beyond that are dense concentrations of ribosomes and organelles (see Tokuyasu et al., 1972, their Figure 11). As the cystic bulge progresses toward the caudal end of the spermatids, it increases in size because of the accumulation of organelles. Eventually the excluded membrane and organelles are pinched off at the caudal end in the waste bag structure. After individualization, each sperm is contained in its own membrane, and only a remnant of the axonemal sheath remains next to the condensed mitochondria (for fine structural analysis of spermatogenesis, see Stanley et al., 1972).

Fabrizio et al. (1998) have recently shown that the individualization complex (IC) within the developing spermatids contains actin as its major cytoskeletal component. They proposed that the IC is a complex of membrane and cytoskeletal proteins. In an effort to identify important components in the individualization process, Fabrizio et al. (1998) examined a number of late male sterile mutations and classified them by distinguishing features of the actin-containing IC. Although this phenotypic analysis has provided a 


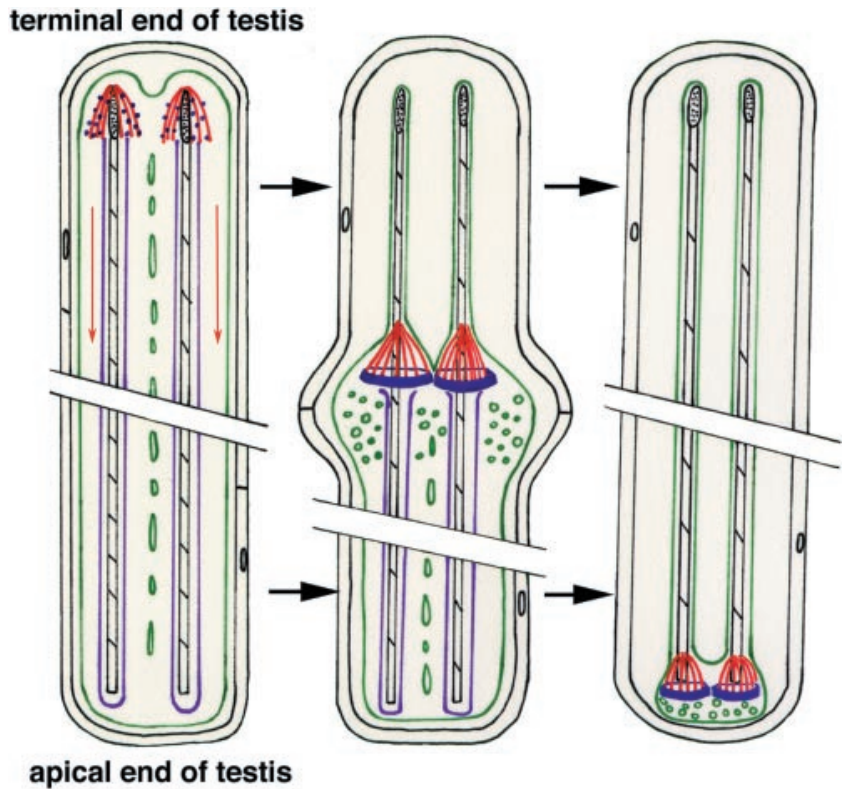

Figure 1. Schematic diagram of individualization. See Figure 5A for schematic diagram of testes and the relationship of this diagram to overall testes organization. Individualization is depicted in this schematic diagram as proceeding chronologically from left to right. A single cyst (cell membrane shown in black) with two representative spermatids is depicted at three different time points. The cysts shown are not drawn to scale and would measure $\sim 2 \mathrm{~mm}$ long inside the wild-type testis. The first cyst depicts the initial assembly of actin around the nuclei (stippled black) and the first appearance of organized 95F myosin. Actin (red) assembles around each spermatid nucleus in the shape of a cone with the advancing edge of the cone toward the tail end of the spermatids. The tail end of the spermatid is toward the apical end of the testes. 95F myosin (blue) localizes in punctate structures along the actin filaments. The actin cones progress from the nuclear region to the end of the tail, in the direction of the red arrows. The spermatid membranes (green) are punctuated by cytoplasmic bridges. The second cyst depicts the actin cones, the spermatid membranes (green) and 95F myosin after progression of the cones away from the nuclei. 95F myosin (blue) forms in a ring at the base of the actin filaments. As the actin-95F myosin complex moves toward the tail end of the spermatids, many vesicles and organelles (green circles) are present in front of the complex. In the wake of the actin-95F myosin complex each sperm is invested in its own membrane (green) and is devoid of vesicles and organelles (with the exception of a single mitochondrion). The axonemal sheath (purple) is also lost during IC progression. Finally, in the third cyst the complex is depicted reaching the end of the axoneme (black-striped structure) just before formation of the waste bag (not drawn to scale), a structure containing the waste organelles and membrane that is pinched off from the base of the cyst. The actin-95F myosin complex, in addition to any other proteins and organelles not incorporated into the mature sperm, is contained in the waste bag and eventually degrades.

wealth of information about the complexity of the individualization process, the only known molecular component of the IC is actin. The work reported here identifies another component of the IC as class VI unconventional myosin.

$95 \mathrm{~F}$ myosin is a member of a large family of unconventional myosins. Currently there are at least 15 classes of unconventional myosins, classified according to similarities within their myosin head domains (for review, see Cope et al., 1996). Several unconventional myosins, including 95F myosin, have been implicated in membrane and organelle trafficking in vitro and in vivo (for reviews, see Titus, 1997; Mermall et al., 1998). 95F myosin is expressed in many tissues throughout the life cycle of the fly. It functions in vivo as an ATP- and actin-dependent transporter in the pregastrulation embryo (Mermall et al., 1994). During this stage of embryogenesis the cells are undergoing rapid rounds of mitosis within a common cytoplasm. 95F myosin is involved in particle movements to and from the invaginating membranes that divide neighboring mitotic apparati in the syncytium. Upon inhibition of 95F myosin with anti95F myosin antibody, these actin-based invaginations fail to form (Mermall and Miller, 1995). Using antisense RNA technology, 95F myosin has been shown to be essential for epithelial morphogenesis throughout development. Gal4upstream activation sequence-driven antisense constructs expressed in different tissues and at different times lead to a variety of phenotypes, including lethality at various developmental stages and abnormalities in the evagination of leg and wing imaginal discs and in the migration of follicle cells in oogenesis (Deng et al., 1999).

We have isolated a partial loss of function mutation in $95 \mathrm{~F}$ myosin, mmw14, which results in male sterility but otherwise does not affect viability or female fertility. We can rescue this mutation with one copy of the 95F myosin heavy chain (MHC) cDNA. In addition, we have determined that this mutation fails to complement another postmeiotic differentiation mutation, jaguar, which was previously isolated in a screen for male sterile mutations (Castrillon et al., 1993). Both of the mutations in $95 \mathrm{~F}$ myosin result in a defect in spermatid individualization. Because of the similarity of effects of 95F myosin loss in the metaphase furrows of the early embryo and during spermatogenesis, we propose that 95F myosin is the motor that is essential for transport and reorganization of membranes at the advancing individualization front.

\section{MATERIALS AND METHODS}

\section{Fly Culture}

All fly stocks were maintained on standard Drosophila cornmeal, agar, and sucrose medium at $25^{\circ} \mathrm{C}$. The jaguar (jar ${ }^{1}$ ) mutant allele, previously described by Castrillon et al. (1993), was obtained from the Bloomington Stock Center (Bloomington, IN). The deletion chromosome, $D f(3 R) S 87-5$, was made by Elizabeth Knust and Jose Campos-Ortega and others (University of Koln) and was obtained from Eric Weischaus (Princeton University). The starting P insert line, C865, came from a Gal4 screen for genes expressed in a subset of follicle cells in oogenesis (Deng et al., 1997, 1999).

\section{Generation of 95F Myosin Deletion Mutant}

The 95F myosin mutation, $m m w 14$, was generated by excision of a $\mathrm{P}$ element insertion, which is located at the $5^{\prime}$ end of the 95F myosin gene on the third chromosome (Figure 2). The starting P insert line, C865, had the genotype $w^{-} / w^{-} ; P\left[w^{+}\right.$, Gal4] $/ P\left[w^{+}\right.$, Gal4]. P element excision was performed by crossing $C 865$ to an endogenous source of transposase, $w^{-} / Y ; P[\Delta 2-3], D r / T M 3, S b$. The dominant marker, $D r$, allows selection for and against the source of transposase. In the F1 generation, $P[\Delta 2-3]$ containing progeny were selected and were crossed to a third chromosome balancer line, $w^{-} / w^{-} ; T M 3, S b / T M 6$, $\mathrm{Tb}$. The resulting progeny were scored for lines that had lost the $\mathrm{P}$ 
element and therefore had white eyes and no longer contained the $P[\Delta 2-3]$. Heterozygous stocks were established by crossing the $P$ excision lines to $w^{-}$; TM3, Sb/TM6, Tb. The potential P excisions were screened for lethality by crossing heterozygous siblings to one another and scoring for the presence of homozygotes by the lack of the marked balancer chromosome.

\section{Generation of the 95F MHC Transgene}

A full-length cDNA encoding the EM3 isoform of 95F MHC was cloned into CaspeR-HS83 downstream of the hs83 promoter. To clone the cDNA into the CaspeR-HS83 vector, first a 950-bp PCR product was generated by using a $5^{\prime}$ primer that added an XhoI site upstream of the initiator methionine and a $3^{\prime}$ primer centered around a unique BamHI site in the middle of the 95F myosin gene. This PCR product was then digested with $\mathrm{XhoI}$ and BamHI and ligated into the XhoI-BamHI-digested CaspeR vector. Next, a BamHI-NotI fragment of 95F MHC was isolated from the cDNA. This fragment contains the rest of the 95F MHC downstream of the BamHI site and includes the 3' UTR. The BamHI-NotI fragment was then ligated into the BamHI/NotI digested CaspeR vector containing the Xho-BamHI fragment of 95F MHC. The CaspeR-HS83 95F MHC cDNA (P[95F MHC]) was injected into homozygous $w^{-}$embryos containing constitutively active transposase, and stable transformants were obtained by standard procedures (Rubin and Spradling, 1982). Adults containing the $\mathrm{P}[95 \mathrm{~F} \mathrm{MHC}]$ were selected for by the presence of an eye color marker $\left(w^{+}\right)$present on CaspeR-HS83.

\section{Rescue of the mmw14 Male Sterile Phenotype}

To test for rescue of the male sterile phenotype of mmw14/ $D f(3 R) S 87-5$ by P[95F MHC], $w$; P[95F MHC]/CyO; mmw14/TM3, $e$, $S b$ males or females were crossed with $w ;+/+; D f(3 R) S 87-5$, e/TM3, $e, S b$ males or females. The resulting male progeny with the genotypes w; P[95F MHC]/+; mmw14/Df(3R)S87-5, e, and $w$; +/+; $m m w 14 / D f(3 R) S 87-5$ were selected and tested for fertility by mating to wild-type virgin females.

\section{Mapping of the Genomic Organization of the 95F Myosin Mutant Alleles}

The P element insertion (C865), which was used to generate mmw14, was mapped to the $5^{\prime}$ end of the $95 \mathrm{~F}$ myosin gene (Deng and Bownes, unpublished results). The P element insertion, $P\left[l a c Z, r y^{+}\right]$ (Castrillon et al., 1993), was mapped relative to the 95F myosin genomic structure using PCR. Two primers, one hybridizing to the end of the P element (CGACGGGACCACCTTATGTTATTTCATC) and one specific for the 95F myosin ATG (ACACACCAGTTGGGTGTCCTCCAACAT), were used to amplify genomic DNA of $j^{1}{ }^{1}$ / jar $^{1}$ adults. The resulting product was $4.5 \mathrm{~kb}$, placing the P element insertion within an intron, $4.5 \mathrm{~kb}$ upstream from the start of translation (see Figure 2). The $m m w 14$ deletion was mapped using Southern blotting with probes from the 95F myosin cDNA and flanking the $P\left[w^{+}\right.$; Gal4] C865 insertion site.

\section{Reverse Transcription (RT)-PCR}

Two to $3 \mu \mathrm{g}$ of total RNA were reverse transcribed by adding $1 \mu \mathrm{l}$ avian myeloblastosis virus reverse transcriptase (Promega, Madison, WI), and the final concentration of the RT buffer was adjusted to $1 \times$. Usually the reaction volume was $50 \mu l$. The reaction was carried out at $48^{\circ} \mathrm{C}$ for $45 \mathrm{~min}$ and stopped at $94^{\circ} \mathrm{C}$ for $2 \mathrm{~min}$.

Three microliters of the RT reaction product were used as the template for PCR. PCR was carried out using a Hybaid (Middlesex, United Kingdom) thermal cycler. The standard reaction volume was $100 \mu \mathrm{l}$ in sterile 0.5-ml tubes. Pharmacia (Piscataway, NJ) dNTPs were used for all reactions. Standard final concentrations of dNTPs and primers were $0.1 \mathrm{mM}$ and $2.5 \mathrm{pmol} / \mu \mathrm{l}$ respectively. The stan- dard PCR buffer used was $50 \mathrm{mM} \mathrm{KCl}, 20 \mathrm{mM}$ Tris-HCl, $\mathrm{pH} 8.3,2$ $\mathrm{mM} \mathrm{MgCl}$ and $1 \mathrm{mg} / \mathrm{ml}$ gelatin.

Primers used for these experiments are p1 (GAGTTCGACTCGACTCATCCAAC), p517 (ATCACGATGACAACTGCGAAC), and p1027 (TAGTGCGATATGTAACCACCGACC).

\section{Antibodies}

The 95F myosin monoclonal antibody 3C7 (Kellerman and Miller, 1992) was diluted 1:20 for Western blots and 1:10 for whole tissue. The anti-tubulin monoclonal antibody DM1A was used at a concentration of 1:10 for Western blots.

\section{Western Analysis}

Either testes or whole animals were homogenized in $2 \times$ Laemmli sample buffer supplemented with $1 \mathrm{mM}$ PMSF and $1 \mathrm{mM}$ EDTA, proteins were separated on a $7.5 \%$ SDS polyacrylamide gel and transferred to a Nitrobind transfer membrane (Micron Separations, Westboro, MA) in glycine buffer (150 mM glycine, $0.02 \%$ SDS, $20 \%$ methanol) at $90 \mathrm{~V}$ for $4 \mathrm{~h}$. The blot was blocked for $1 \mathrm{~h}$ in $5 \%$ nonfat dry milk in TBST (Tris-buffered saline [TBS; $25 \mathrm{mM}$ Tris, $135 \mathrm{mM}$ $\mathrm{NaCl}, 2.6 \mathrm{mM} \mathrm{KCl}$ ], pH 8, and 0.05\% Tween 20 [Sigma, St. Louis, $\mathrm{MO})$ and incubated with primary antibody diluted in $5 \%$ nonfat dry milk for $2 \mathrm{~h}$ at room temperature. The blot was washed four times for 15 min each with TBST, incubated with Immunopure goat anti-mouse HRP (Pierce, Rockford, IL) at 1:10,000 for $1 \mathrm{~h}$, and washed four times for $15 \mathrm{~min}$ each with TBST. Detection was performed using enhanced chemiluminescence (Super Signal Substrate; Pierce). The protein standards were broad-range standards from Bio-Rad (Hercules, CA; 200, 116.2, 97.4, 66.2, 45, 31, 21.5, 14.4 and $6.5 \mathrm{kDa})$.

\section{Immunofluorescent Staining}

Testes were prepared for immunofluorescent staining by the method of Hime et al. (1996). Testes were dissected in testes buffer 1 ( $15 \mathrm{mM}$ potassium phosphate, $\mathrm{pH} 6.7,80 \mathrm{mM} \mathrm{KCl}, 16 \mathrm{mM} \mathrm{NaCl}$, $5 \mathrm{mM} \mathrm{MgCl}$, and $1 \%$ polyethylene glycol 8000 ), transferred to a poly-L-lysine-coated slide, and squashed with a siliconized coverslip. The slide and coverslip were frozen in liquid nitrogen, the coverslip was removed with a razor blade, and the slide was placed in $95 \%$ ethanol on dry ice for $10 \mathrm{~min}$. The slide was drained, fixed in $4 \%$ formaldehyde (EM Science, Gibbstown, NJ) in PBS for $7 \mathrm{~min}$, washed twice for $15 \mathrm{~min}$ in PBS $+0.3 \%$ Triton X-100 and $0.3 \%$ sodium deoxycholate, and rinsed in PBT (PBS + 0.1\% Triton X-100). The fixed testes were then blocked with PBTB (PBS + 0.1\% Triton X-100 + 3\% BSA) for $30 \mathrm{~min}$, incubated with primary antibody (anti-95F myosin monoclonal antibody, 3C7, at 1:10) for $2 \mathrm{~h}$ at room temperature, and rinsed four times for $15 \mathrm{~min}$ with PBTB. Testes were incubated with secondary antibody (goat anti-mouse Oregon Green; Molecular Probes, Eugene, OR) and/or rhodaminephalloidin (Sigma) in PBTB for $1 \mathrm{~h}$ at room temperature and then rinsed one time for $15 \mathrm{~min}$ in PBTB. For staining DNA, the testes were incubated with $1 \mu \mathrm{g} / \mathrm{ml}$ DAPI in PBTB for $10 \mathrm{~min}$ and then rinsed two times with PBTB. Testes were mounted in mounting medium ( $10 \%$ glycerol $+1 \mathrm{mg} / \mathrm{ml} p$-phenylenediamine).

\section{Cytological Analysis}

To assay for spermatid motility, testes were dissected from newly eclosed Oregon-R, mmw14/Df(3R)S87-5 or jar ${ }^{1} /$ jar $^{1}$ males in Drosophila Ringer's solution with 1,4-piperazinediethanesulfonic acid according to the method of Castrillon et al. (1993). Dissected testes were squashed under coverslips and examined immediately by phase-contrast microscopy. 


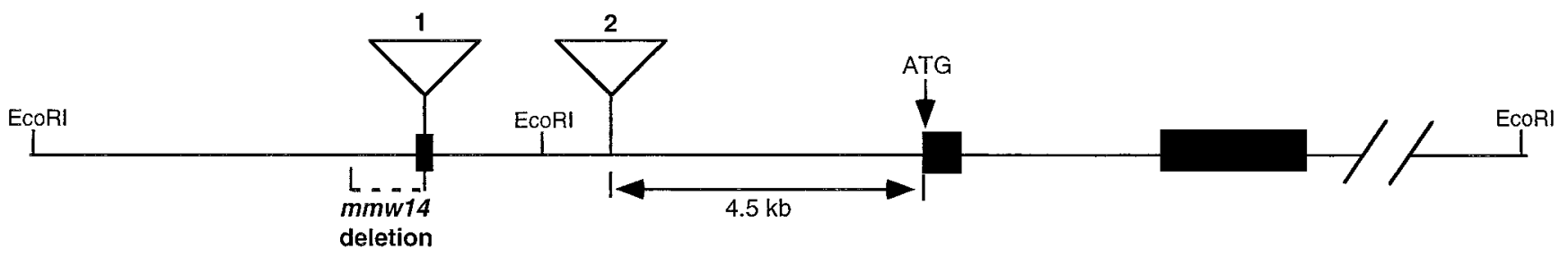

Figure 2. $95 \mathrm{~F}$ myosin genomic structure. The genomic structure of $95 \mathrm{~F}$ myosin shows the relative position of $\mathrm{P}$ element insertions and the mmw14 deletion. 95F myosin exons are shown in black. $\mathrm{P}$ element 1 is $\mathrm{P}\left[\mathrm{w}^{+}\right.$; Gal4], which is inserted $5^{\prime}$ of the first exon yielding the C865 line. Imprecise excision of this P element resulted in a 1-kb deletion, $m m w 14$, which extends from the P[w $w^{+}$; Gal4] insertion site into the flanking DNA. Insertion of P element 2, P[lacZ, ry ${ }^{+}$, yields the jaguar mutation. Based on PCR analysis with primers to exon 2 of $95 \mathrm{~F}$ myosin and the $\mathrm{P}$ end, the distance of $\mathrm{P}$ element 2 is $\sim 4.5 \mathrm{~kb}$ from the initiator methionine of $95 \mathrm{~F}$ myosin (our unpublished results).

\section{Scoring ICs}

Testes were dissected from 1- to 2-d-old males of the indicated genotype and prepared as above for immunofluorescent microscopy. The fixed testes were stained with both rhodamine-conjugated phalloidin (Sigma) and DAPI. In general the scoring of the ICs was based on the method of Fabrizio et al. (1998), but a detailed description follows. For each genotype (wild-type, jar $^{1} /$ $j_{a r^{1}}$ or jarm $/ D f(3 R) S 87-5$ ), several (either 8 or 10) testes were examined. First the total number of ICs per testis was counted on the basis of staining with rhodamine-conjugated phalloidin. Then for each IC it was determined whether the IC colocalized with nuclei by examining the IC for colocalizing DAPI staining. Each IC was carefully observed to determine whether the actin cones were in register with one another. If they were in register they were counted as "intact." If they were out of register in any way they were counted as "disrupted." If rhodamine-phalloidin staining did not coincide with DAPI staining, it was assumed that the IC had progressed away from the nuclei; therefore the IC was counted as "progressed." The progressed ICs were also carefully observed to determine whether the actin cones were in or out of register and were counted as either intact or disrupted accordingly.

\section{RESULTS}

\section{$P$ Element Insertion in the 95F Myosin Gene Causes Male Sterility}

The 95F myosin mutation, mmw14, was isolated by imprecise excision of a P element, which mapped to the 5 ' end of the 95F myosin gene (Figure 2; Deng and Bownes, unpublished results). The initial P element insertion line, C865, did not exhibit a phenotype in homozygous mutant lines. We excised the P element to create a mutation in 95F myosin. Several lines that show deletion of either $5^{\prime}$ or $3^{\prime}$ DNA at the $P[$ Gal4] insertion site were obtained. One of these lines, $m m w 14$, has a portion of the first exon removed (Figures 2 and $3, \mathrm{~A}$ and B). mmw14 heterozygous with the deficiency, $D f(3 R) S 87-5$, which removes the 95F myosin gene (Kellerman and Miller, 1992), results in viable adults. Fertility of females is not affected in mmw14/Df(3R)S87-5 animals. However, male $m m w 14 / D f(3 R) S 87-5$ animals are sterile. The male sterility phenotype is also observed in another deletion mutant, mfw98, in which $2 \mathrm{~kb}$ of $5^{\prime}$ genomic sequence flanking the P[Gal4] insertion site in C865 were deleted. mmw14/ mfw98 males also exhibit a male sterile phenotype, indicating that both excision mutants are in the same complementation group. The male sterile phenotype is due to a partial loss of $95 \mathrm{~F}$ myosin function in these flies (see below).
Before this work Castrillon et al. (1993) had performed a P element insertion screen for genes that affected male fertility. They recovered a line with a $\mathrm{P}$ element insertion in the $95 \mathrm{~F}$ region, which they called jaguar. When either homozygous or in trans with $D f(3 R) S 87-5$, jaguar results in male sterility. At the time they were unable to determine the identity of the gene or genes affected by this insert. Analysis of DNA that flanks the insertion did not yield any known genes, and the $P$ element was believed to reside within an intron (Wasserman, personal communication). Subsequently, we determined by complementation analysis that mmw14 and jaguar are allelic to one another: the $m m w 14 / j a r^{1}$ transheterozygote is male sterile. This complementation analysis demonstrates that $m m w 14$ and $j a r^{1}$ are mutations in the same gene. Because jar mutations were the first mutations isolated, we will refer to all subsequent mutations in this gene as alleles of jar. Therefore, mmw14 has been renamed jarmmw14 in keeping with the conventions of Drosophila nomenclature.

We mapped the $\mathrm{P}$ element insertion in $j a r^{1}$ to the first intron of 95F myosin (Figure 2) using PCR with primers at the $\mathrm{P}$ element end and within the 95F myosin cDNA (see MATERIALS AND METHODS). We and others (Wasserman, unpublished observations) have determined by excision of the P element that it is responsible for the male sterile mutation. We introduced an endogenous source of transposase, $\Delta 2-3$, into the $j a r^{1} / j a r^{1}$ background and scored the resulting offspring for the loss of the P element. Of the 42 independent excisions we recovered, none was lethal, 14 were male sterile, and 28 were fertile. Because we are able to revert the male sterile phenotype to fertility by loss of the $\mathrm{P}$ element, we conclude that the P element insertion is responsible for the male sterility.

\section{F Myosin cDNA Rescues the Sterile Phenotype of mmw14}

To determine whether the jarmmw14 male sterile phenotype resulted from a reduction in 95F myosin function, we tested the ability of ectopically expressed 95F MHC to rescue the jarmmw14 fertility defect. Transgenic animals that contain a 95F MHC cDNA driven by hs83, a promoter known to be active in the Drosophila germ line, were generated by $\mathrm{P}$ element-mediated transformation, and the transgene was crossed into the mutant background (see MATERIALS AND METHODS). Twenty-four hemizygous jarmmw14 / $D f(3 R) S 87-5$ males that contained one copy of the 95F MHC transgene were mated with virgin females. Of these 24 


\section{A}

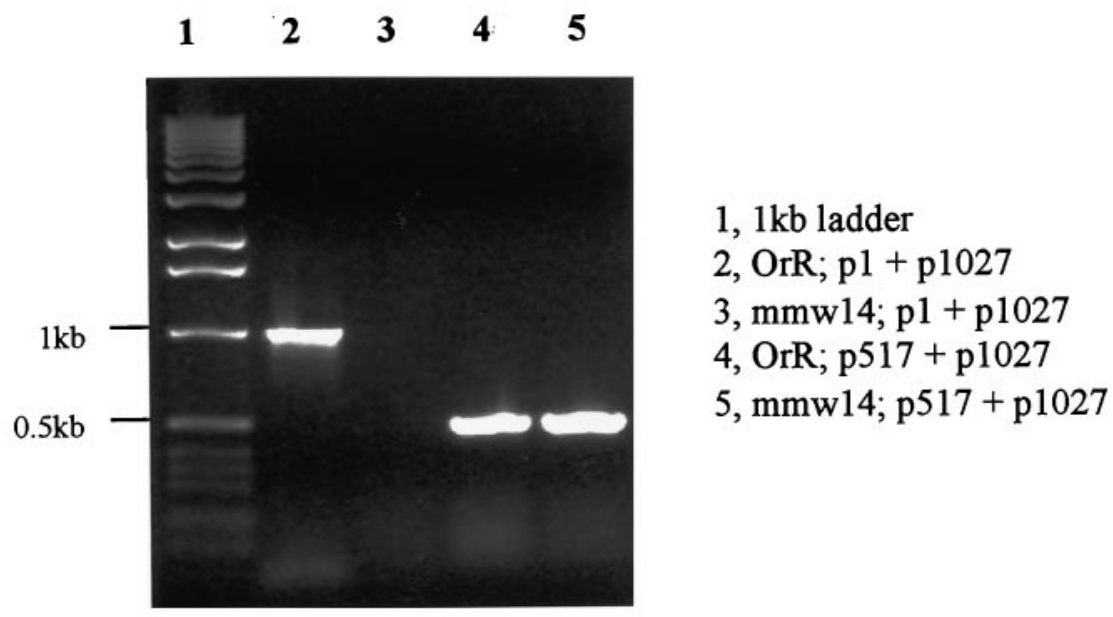

Figure 3. RT-PCR to detect transcription

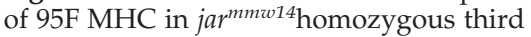
instar larvae. (A) p1027 was used as the reverse transcription primer in lanes 2 and 3. Amplification primer pairs are listed on the right. No products were present in the jarmmo14homozygous third instar larvae when primer pair p1 and p1027 was used (lane 3), whereas the control wild-type (Oregon-R) larvae had a 1-kb product (lane 2). This suggests that no transcripts containing the first exon are present. Truncation of this exon does not disrupt transcription of the 95F MHC gene, because a $0.5-\mathrm{kb}$ band is observed when using the primer pair p517 and p1027 to amplify the reverse transcriptional products (lane 5). The 0.5-kb band (lanes 4 and 5) was not due to genomic DNA contamination, because two bands were detected when the genomic DNA was used as the template, and p517 and p1027 were used as amplification primers (our unpublished results). (B) A schematic diagram shows the location of the 95F MHC primers used in this experiment.
B

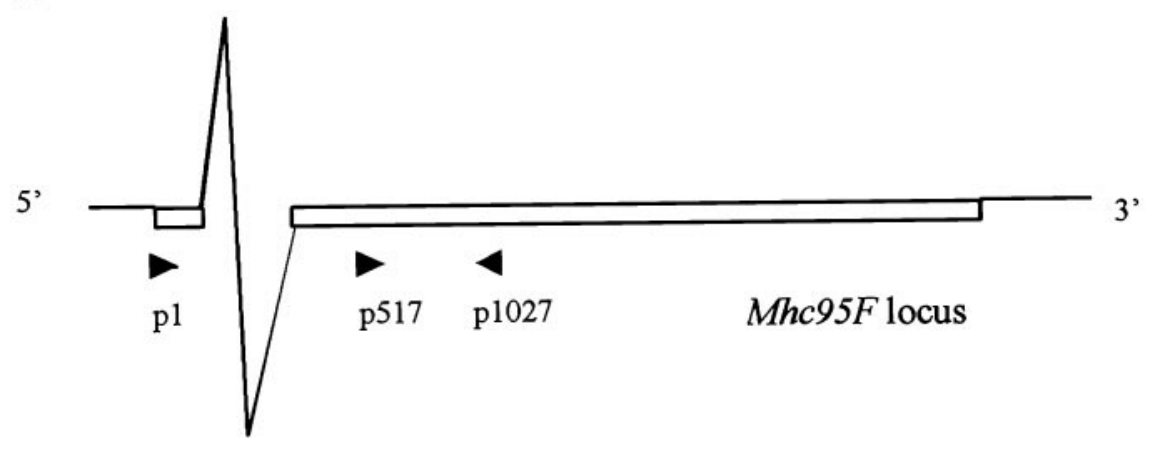

males, $83 \%$ were fertile and produced viable offspring. In contrast, $100 \%$ (20 of 20) of the hemizygous males that lacked the $95 \mathrm{~F} \mathrm{MHC}$ transgene were infertile and produced no offspring. Therefore, the $95 \mathrm{~F}$ MHC cDNA is sufficient to rescue the jar ${ }^{m m w 14}$ male sterile phenotype, supporting our molecular evidence that jarmmw14 is a mutation in the $95 \mathrm{~F}$ MHC.

\section{jar1 $^{1}$ and jar ${ }^{\text {mmw14 }}$ Are Partial Loss-of-Function Mutations}

The jarmmw14 deletion does not prevent expression of $95 \mathrm{~F}$ myosin in all tissues. To demonstrate this we performed RT-PCR (Figure 3) with the primer pairs p1 and p1027. p1 is at the transcription start site in the first exon, whereas p1027 is in the second exon. In wild-type third instar larvae, the p1 and p1027 primer pair yielded a band of $\sim 1 \mathrm{~kb}$ (Figure 3A, lane 2). However, in the jar ${ }^{m m w 14}$ homozygous larvae this band was missing (Figure 3A, lane 3). RT-PCR with p517, a primer which is $3^{\prime}$ of the first exon, and p1027 yields a band of $500 \mathrm{bp}$ in both the wild-type and jarmmw14 larvae (Figure 3A, lanes 4 and 5). This suggests that a second transcription start site exists, and the deletion in jarmmw14, which only deletes untranslated sequences, does not prevent expression of this gene in whole animals.

Animals homozygous for jarmmw14 die as late as third instar larvae. We attribute this lethality to a mutation at another chromosomal region, because jarmmw14 / Df(3R)S87-5 animals are viable and exhibit an identical male sterile phenotype as the jar ${ }^{1} /$ jar $^{1}$ mutant (see above). Because $j a r^{1} /$ jar $^{1}$, $j a r^{1} / j a r^{m m w 14}$, and jarmmw14 / Df(3R)S87-5 have the same phenotype, i.e. male sterility, and jarmmw14 / $f(3 R) S 87-5$ can be rescued by the $95 \mathrm{~F}$ MHC transgene, the unlinked homozygous lethality of the $m m w 14$ allele is irrelevant to our analysis. To date none of the deletion lines we generated is a null mutation in $95 \mathrm{~F}$ myosin.

Because jarmmw14 animals still produce 95F myosin transcripts, we suspected that this allele might affect regulation of the gene. To determine whether expression of $95 \mathrm{~F}$ myosin protein was affected in testes, we analyzed the carcass and testes from mutant animals by immunoblotting for 95F myosin (Figure 4). In wild-type adults $95 \mathrm{~F}$ myosin is present as a $140-k D a$ protein (Kellerman and Miller, 1992). When one copy of the deficiency, $D f(3 R) S 87-5$, is introduced into the wild-type background, the amount of $95 \mathrm{~F}$ myosin is reduced (lane 2 ). In jar ${ }^{m m w 14}$ / Df(3R)S87-5 (lane 4) or jar ${ }^{1} /$ jar $^{1}$ (lane 6) adult carcass, 95F myosin is present in amounts similar to that seen in $D f(3 R) S 87-5 /+$ animals. Thus, in these mutant animals $95 \mathrm{~F}$ myosin that is of normal size is expressed in most tissues.

In contrast, 95F myosin expression in testes is affected by these mutations. 95F myosin is present in substantial amounts in the testes of wild-type (Figure 4, lane 7) and $D f(3 R) S 87-5 /+$ (lane 8). However, 95F myosin protein is 


\section{3}

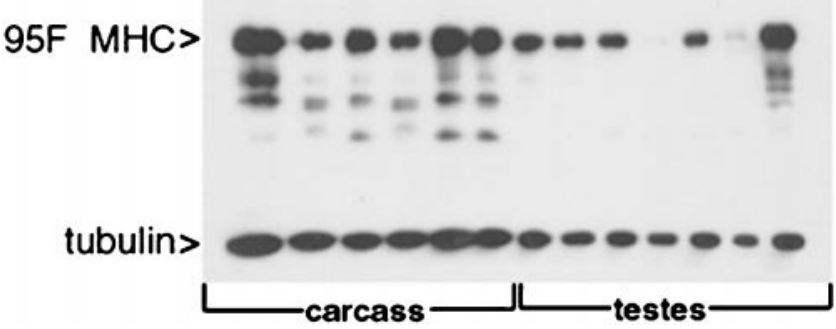

Figure 4. Western analysis of 95F myosin mutants. Protein was prepared either from carcass (lanes 1-6) or testes (lanes 7-13) of animals of the genotypes indicated. The top half of the blot was probed with anti-95F myosin monoclonal antibody (3C7), and the bottom half of the blot was probed with anti-tubulin monoclonal antibody. Lane 1, wild type; lane 2, Df(3R)S87-5/TM6; lane 3, jarmmw14/TM6, lane 4, jarmmw14/Df(3R)S87-5; lane 5, jar1/TM3; lane 6, jar $^{1} /$ jar $^{1}$; lane 7 , wild type; lane 8, Df(3R)S87-5/TM6; lane 9, jarmmw14/ TM6, lane 10, jarmmw14/Df(3R)S87-5; lane 11, jar ${ }^{1} / T M 3$; lane 12, jar ${ }^{1}$ / jar $^{1}$; lane 13, P[95F MHC]; jarmmw14/Df(3R)S87-5. Asterisks indicate the lanes that show protein from mutant animals. 95F myosin is present in all carcass lanes (lanes 1-6) but is absent in jarmmw14 $D f(3 R) S 87-5$ testes (lane 10) and significantly reduced in $\mathrm{jar}^{1} / \mathrm{jar}^{1}$ testes (lane 12). The P[95F MHC]-containing animals exhibit high levels of expression in testes in the mutant background, jarmmw14/ $D f(3 R) S 87-5$ (lane 13). The bands present beneath the 140-kDa 95F myosin band are proteolyzed forms of $95 \mathrm{~F}$ myosin also recognized by $\mathrm{mAb} 3 \mathrm{C}$. The tubulin staining serves as a loading control.

either completely absent (jarmmw14 / $D f(3 R) S 87-5$; lane 10) or significantly reduced in amount (jar $/ / j a r^{1}$, lane 12) in testes from the mutants. 95F myosin is also present in homozygous jar ${ }^{m m w 14}$ mutant larvae (our unpublished results). The presence of the 95F myosin cDNA transgene results in elevated expression of $95 \mathrm{~F}$ myosin in the testes (P[95F MHC];

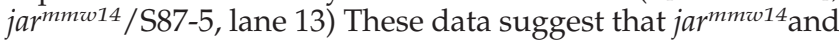
$\mathrm{jar}^{1}$ are partial loss-of-function mutations in the $95 \mathrm{~F}$ myosin heavy chain. Expression of the gene is not affected in most adult tissues but is reduced or absent in testes.

\section{Gross Morphology of Mutant Testes Is Normal}

The cause of the male sterile phenotype was investigated by detailed examination of testes from mutant animals. To determine whether the size, overall gross morphology of the testes, or meiotic and mitotic stages were affected, we examined mutant and wild-type testes from newly eclosed males stained with DAPI to visualize nuclei. The testes is organized as a spiral structure with the early stages of spermatogenesis at the apical end (Figure 5A). The heads of the elongated spermatids are near the terminal end of the spiral, and individualization proceeds toward the apical end. A single individualizing cyst of 64 haploid spermatids is highlighted in red.

We did not observe any differences in size or overall morphology between wild-type and jarmmw14 /Df(3R)S87-5 testes (Figure 5, B and C). The jar $/ \mathrm{jar}^{1}$ testes were similar (our unpublished results). In addition, the early meiotic and mitotic stages appear normal (our unpublished results).
However, there were several differences readily apparent in organization of the spermatid nuclear clusters. In wild type, the nuclear clusters are brightly stained and reside in the terminal third of the testes near the seminal vesicle (Figure 5B). In jarmmw14/Df(3R)S87-5, some nuclear clusters are smaller (and therefore not as brightly stained) and are present closer to the apical end of the testes. We postulate that the presence of bundles in the more apical region results from an accumulation of mutant spermatids in the testes because they are never released into the seminal vesicle. We never observed sperm in the seminal vesicle of the jarmmw14 / $D f(3 R) S 87-5$ testes (our unpublished results).

\section{jarmmw14 and jar ${ }^{1}$ Sperm Exhibit Defects in Sperm Motility}

The lack of sperm in the jarmmw14 seminal vesicles indicated to us that there could be a defect either in sperm morphology or sperm motility. To determine the basis for this phenotype, mutant testes were squashed and prepared as described in Fabrizio et al. (1998). Wild-type sperm that are properly individualized exhibit a rapid sinusoidal movement in the squash preparation. jarmmw14/Df(3R)S87-5 sperm completely lack motility, and $\mathrm{jar}^{1} / \mathrm{jar}^{1}$ sperm exhibit a slower sinusoidal motion as compared with wild type. When examined by phase-contrast light microscopy the mutant sperm of either genotype does not exhibit any gross morphological defects, such as blebbing of the cytoplasm (Fabrizio et al., 1998). The motility defect suggested that 95F myosin mutations might affect individualization.

\section{F Myosin Is a Component of the IC}

To determine whether individualization was affected, we stained testes with rhodamine-phalloidin, anti-95F myosin monoclonal antibody (3C7), and DAPI to examine IC organization in detail. We first examined wild-type testes to determine the normal subcellular localization of $95 \mathrm{~F}$ myosin. During individualization a cytoskeletal complex called the IC assembles around the clustered nuclei and travels the length of the testes toward the tips of the tails (Figure 1). The IC is made up of 64 actin-rich cones, one of which surrounds each nucleus. The base of the cones leads as the IC travels away from the nuclei. The plasma membrane that was shared by all the spermatids in the cyst becomes resolved into a single membrane for each sperm as the IC progresses. Finally, the IC terminates in the waste bag, which is eventually pinched off from the cyst.

$95 \mathrm{~F}$ myosin is present in a subcortical distribution in the spermatocytes before meiosis (our unpublished results). After elongation, as the IC assembles, 95F myosin localizes in the region of the nuclear clusters in a particulate distribution (Figure 6A). After formation of the IC (Figure 6E), 95F myosin tightly localizes in a band of staining at the base of each investment cone (Figure 6D). A tracing of the actin cones in Figure $6 \mathrm{E}$, with the limits of the cyst and an arrow indicating the direction of IC movement, is shown for orientation in Figure 6F. Based on our observations, it is not clear whether 95F myosin assembles into a ring before or after the actin cones initiate movement. Investigation of the dynamics of this structure would require live imaging of ICs. The band of 95F myosin staining remains at the leading edge of actin cones as the IC traverses the length of the testis (Figure 6G). 


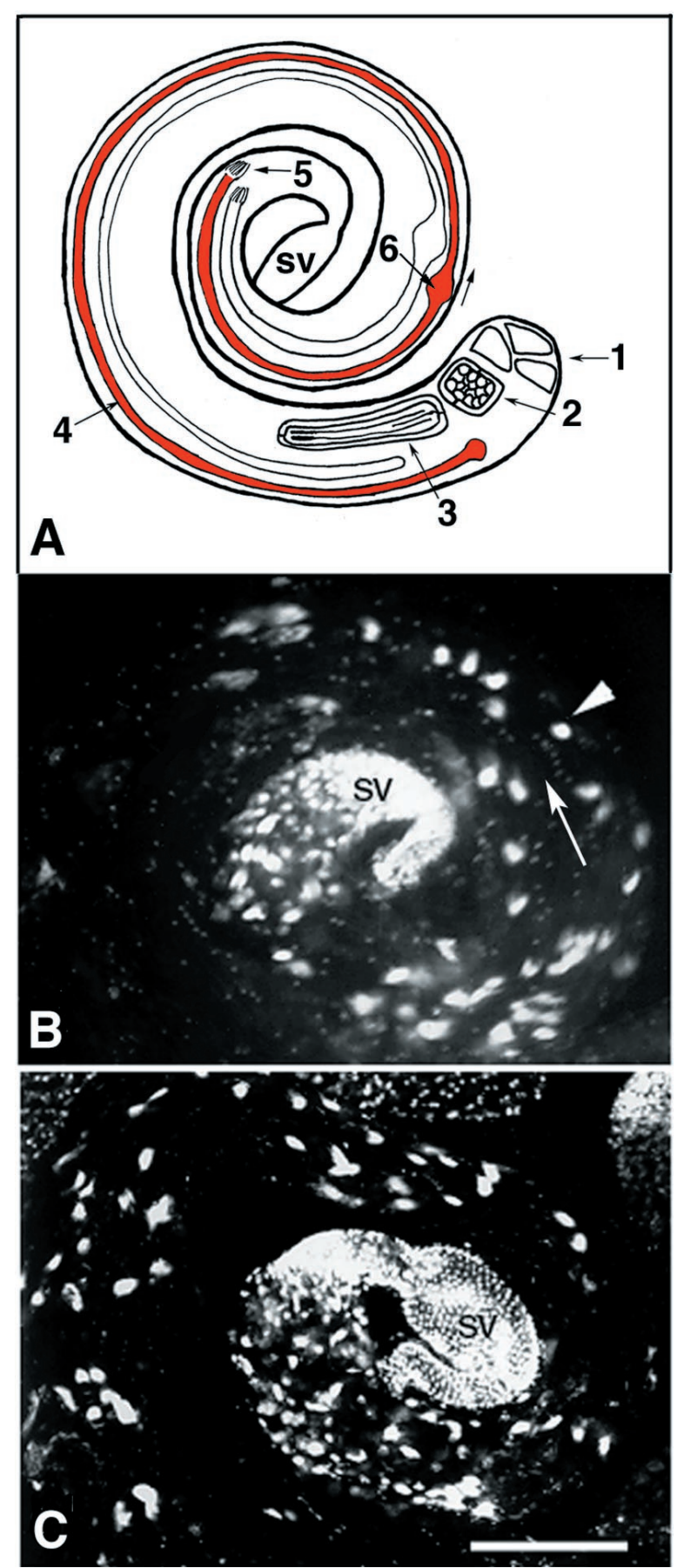

Figure 5. Testis morphology in 95F myosin mutants. Schematic diagram of single wild-type testis indicates the key features in maturation of the male germ cells. Drosophila testes appear as spiral structures. The elongated spermatids are oriented with the caudal end of the spermatid tails toward the apical end and the spermatid heads toward the seminal vesicle (sv; terminal end). A single cyst of 64 spermatids undergoing individualization is shaded red. (1) Hub of stem cells at the apical end from which the germ cells and cyst cells are derived. (2) Cyst of 64 haploid germ cells before elongation. (3) Cyst of 64 haploid germ cells undergoing elongation, interconnected by cytoplasmic bridges. (4) Single cyst, which has undergone
In fact, 95F myosin appears to become more concentrated at this site as the complex moves. Upon completion of individualization, the complex, its proteins, and the membranous material that has been excluded from the spermatid terminate in the waste bag structure. This structure is eventually pinched off at the base of each spermatid. 95F myosin continues to localize in a ring-like structure (Figure 6G), even as the actin filaments appear to break down in the waste bag (Figure $6 \mathrm{H}$ ). The ring-like nature of the staining is seen at high magnification in a more oblique optical section (Figure 6G, inset).

To determine whether 95F myosin-containing rings overlap with actin-containing investment cones, we overlaid the distributions of 95F myosin and actin (Figure 7). It is clear that $95 \mathrm{~F}$ myosin overlaps the actin distribution in a thin band at the leading edge of the investment cone. This particular IC is flattened so that the individual cones are more clearly visualized. The outline of the cystic bulge is apparent in front of the IC.

\section{IC Organization Is Aberrant in 95F Myosin Mutants}

In the jar ${ }^{m m w 14} / D f(3 R) S 87-5$ testes, 95F myosin staining is absent from the spermatocytes and spermatids but is present in somatic cells, such as the cells that constitute the seminal vesicle (our unpublished results). The actin filaments of the IC assemble around each nuclear bundle (Figure 8A) but often the nuclei (Figure 8B) are not aligned in register as in wild type (Figure 6, B,C, and E). The majority of the ICs that colocalize with the nuclear bundles have their component actin cones out of register and thus are of abnormal morphology. These are called disrupted ICs. In $j a r^{1} / \mathrm{jar}^{1}$ testes, occasionally 95F myosin is present in a particulate manner near the nuclei, but more often $95 \mathrm{~F}$ myosin staining is absent in the germ cells, as in the jarmmw14 /Df(3R)S87-5 testes. Although the actin assembles around the nuclei, the individual cones of the ICs in $j a r^{1} / j^{1} r^{1}$ spermatids also are not aligned properly (Figure 8, C and D). Progressed ICs that have their component cones out of register and do not contain 95F myosin are frequently observed (Figure 8E).

To determine whether the rescue of the mutant male sterile phenotype by the $95 \mathrm{~F}$ MHC cDNA restored proper localization of $95 \mathrm{~F}$ myosin in the spermatids, we stained the

Figure 5 (cont.) elongation (red) and is in the process of individualizing. The IC moves in the direction of the arrow. (5) Terminal end of the cyst where the sperm and elongating spermatid nuclei reside. (6) Cystic bulge of the individualizing spermatids. Wild-type (B) and mutant (C) testes stained with DAPI to visualize DNA have similar overall morphology. Note that the region corresponding to the apical portion in which stem cell divisions are occurring is not shown in the micrographs. Large spots of DAPI staining material are bundles of nuclei of either individualized sperm or spermatids that have yet to complete individualization. A single bundle of 64 spermatids (arrowhead) is indicated. In wild type (B) the nuclear clusters are localized to the terminal third of the testes, whereas in the mutant $(\mathrm{C})$ the nuclear clusters are present more toward the apical end of the testes. The arrow indicates the direction in which the ICs travel during individualization. Single bundles are shown in higher magnification in Figures 6 and 7. sv, seminal vesicle. Bar, $10 \mu \mathrm{m}$. 

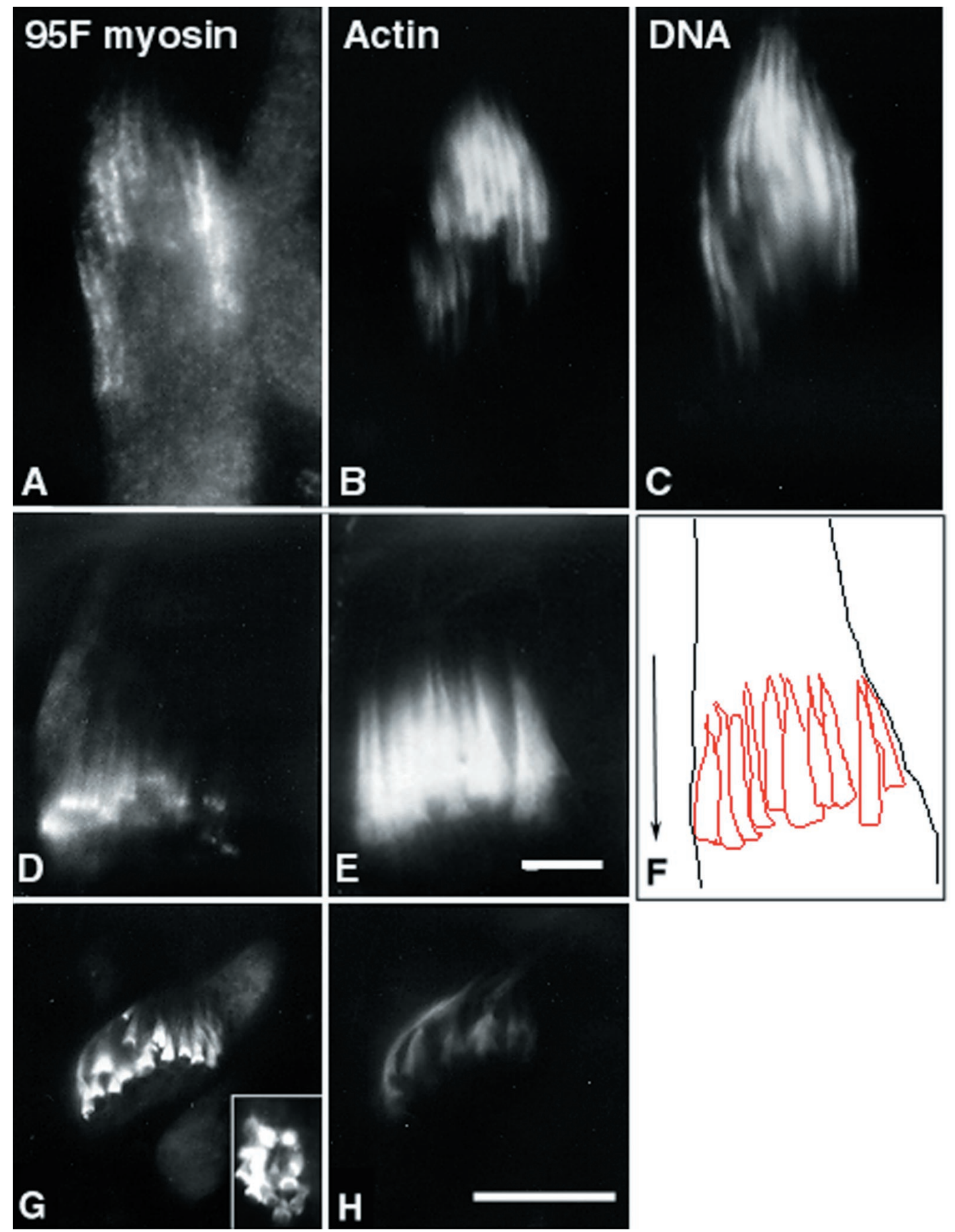

Figure 6. Localization of 95F myosin, actin, and DNA in individualizing spermatids. Each panel is an enlargement of a single cyst of 64 spermatids. At the terminal end of a cyst from a jar/TM3 testes (indistinguishable from wild type), the IC (actin staining; B) assembles around a nuclear bundle. The actin filaments form a cone around each nucleus (C) with a denser distribution at the base of the cone (in the direction of individualization) and the tip of the cone trailing off in the terminal direction. The nuclei are slender and elongated. Just before movement of the IC, 95F myosin (A) localizes along each actin bundle in large particles. In ICs that have moved away from the nuclear bundles (D and E), 95F myosin localizes in distinct bands at the front of the advancing actin cones. The focal planes in D and E are different to optimize the visualization of the actin cones and 95F myosin rings. The actin cones and the outline of the cyst were traced (F) to better illustrate the organization of the IC relative to the cyst. The direction of movement of the advancing IC is shown by the arrow. Actin $(\mathrm{H})$ and 95F myosin $(\mathrm{G})$ are shown in the waste bag. The organization of $95 \mathrm{~F}$ myosin and actin are maintained in the waste bag, although actin appears to degrade before 95F myosin. The inset in $\mathrm{G}$ is a higher-magnification view of an oblique optical section to better illustrate the 95F myosin "rings." Bars, $1 \mu \mathrm{m}$. 

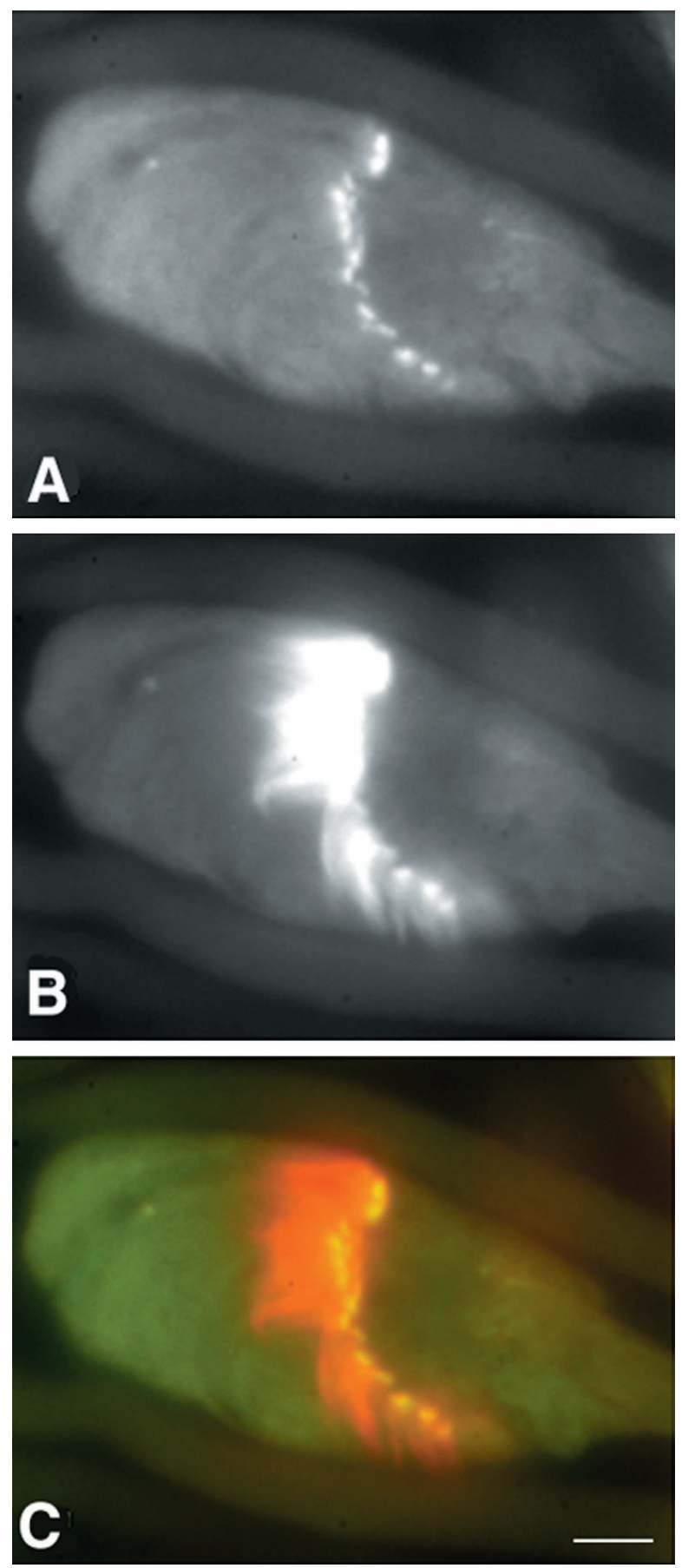

Figure 7. Actin and 95F myosin localization in a progressed IC. (A) Actin, (B) 95F myosin, and (C) overlay of actin (red) and 95F myosin (green). 95F myosin distribution overlaps with actin in a thin band at the base of the investment cones (yellow). Bar, $1 \mu \mathrm{m}$.

P[95F MHC ]; jarmmw14 / Df(3R)S87-5 testes with anti-95F myosin and rhodamine-phalloidin. 95F myosin localized in a band at the advancing front of the investment cone similar to wild type (our unpublished results).

\section{Individualization Is Disrupted in the Mutants}

To quantitate IC behavior and morphology in mutant animals compared with wild type, we fixed and stained wildtype, jarmmw14 $/ D f(3 R) S 87-5$ and jar $^{1} /$ jar $^{1}$ testes from newly eclosed flies. We examined 8 wild-type testes and 10 testes each for jarmmw14/Df(3R)S87-5 and jar ${ }^{1} / j a r^{1}$. We selected several parameters by which to score the ICs, following the convention of Fabrizio et al. (1998) (for further details, see Materials and Methods). We counted the number of rhodamine-phalloidin-stained ICs in each testis and of those determined the number associated with DAPI stained nuclear bundles. We also counted the number of ICs that associated with nuclear bundles but had their component investment cones out of register (disrupted; see Figure 8A for example of a disrupted IC). Finally, we counted the number of ICs that did not colocalize with DAPI (progressed). We noted for the ICs that had progressed whether all the investment cones were closely apposed in register or disrupted. An example of an intact, progressed IC is shown in Figure 6E, whereas a disrupted, progressed IC is shown in Figure 8E. The results of the quantitation are summarized in Figure 9.

Both classes of mutant testes have fewer ICs per testis than wild type. The average number of ICs in wild-type testes is 22, whereas in jarmmw14 and jar ${ }^{1}$, an average of 10 and 14 ICs, respectively, is observed. In wild-type testes most ICs were intact and either associated with nuclei $(62 \%)$ or progressed away from nuclei $(34 \%)$. ICs either at the nuclei or the terminal structure, the waste bag, are most common, suggesting that the ICs progress relatively quickly from the head to the end of the tail. There were very few ICs in wild type that were disrupted and colocalized with nuclei $(3 \%)$ or were disrupted and progressed (0.3\%). Both jarmmw14/ $D f(3 R) S 87-5$ and $j^{1} r^{1} / j_{a r}{ }^{1}$ testes displayed significantly fewer intact ICs either colocalizing with the nuclear bundles or progressed compared with wild-type controls. The majority of ICs in the mutants were either disrupted and associated with nuclei $\left(55 \%\right.$, jar ${ }^{m m w 14}$ and $43 \%$, jar $\left.{ }^{1}\right)$ or disrupted and progressed $\left(24 \%\right.$, jarmmw14 and $42 \%$, jar $\left.{ }^{1}\right)$.

\section{DISCUSSION}

We have identified three independent partial loss-of-function mutations in a class VI unconventional myosin, 95F myosin, that result in male sterility because of a defect in spermatid individualization. Although several groups have suggested possible mechanisms for spermatid individualization (Tokuyasu et al., 1972; Fuller, 1993; Fabrizio et al., 1998), the specific molecules involved in this process, with the exception of actin and clathrin, have not been identified. Our phenotypic analysis of the 95F myosin mutants leads us to suggest that 95F myosin is a motor component of the IC, which may play a role in the reorganization of membrane required for individualization (see below).

$95 \mathrm{~F}$ myosin is not required for the initial assembly of actin into the IC, because in mutant animals actin initially assembles normally. After actin is recruited to the complex but before any movement, 95F myosin localizes in particulate structures along the length of the nucleus. These particulate structures are reminiscent of $95 \mathrm{~F}$ myosin localization in other tissues (Kellerman and Miller, 1992; Mermall and Miller, 1995; Lantz and Miller, 1998) and may represent vesicular or organellar cargo. In the absence of 95F myosin, 


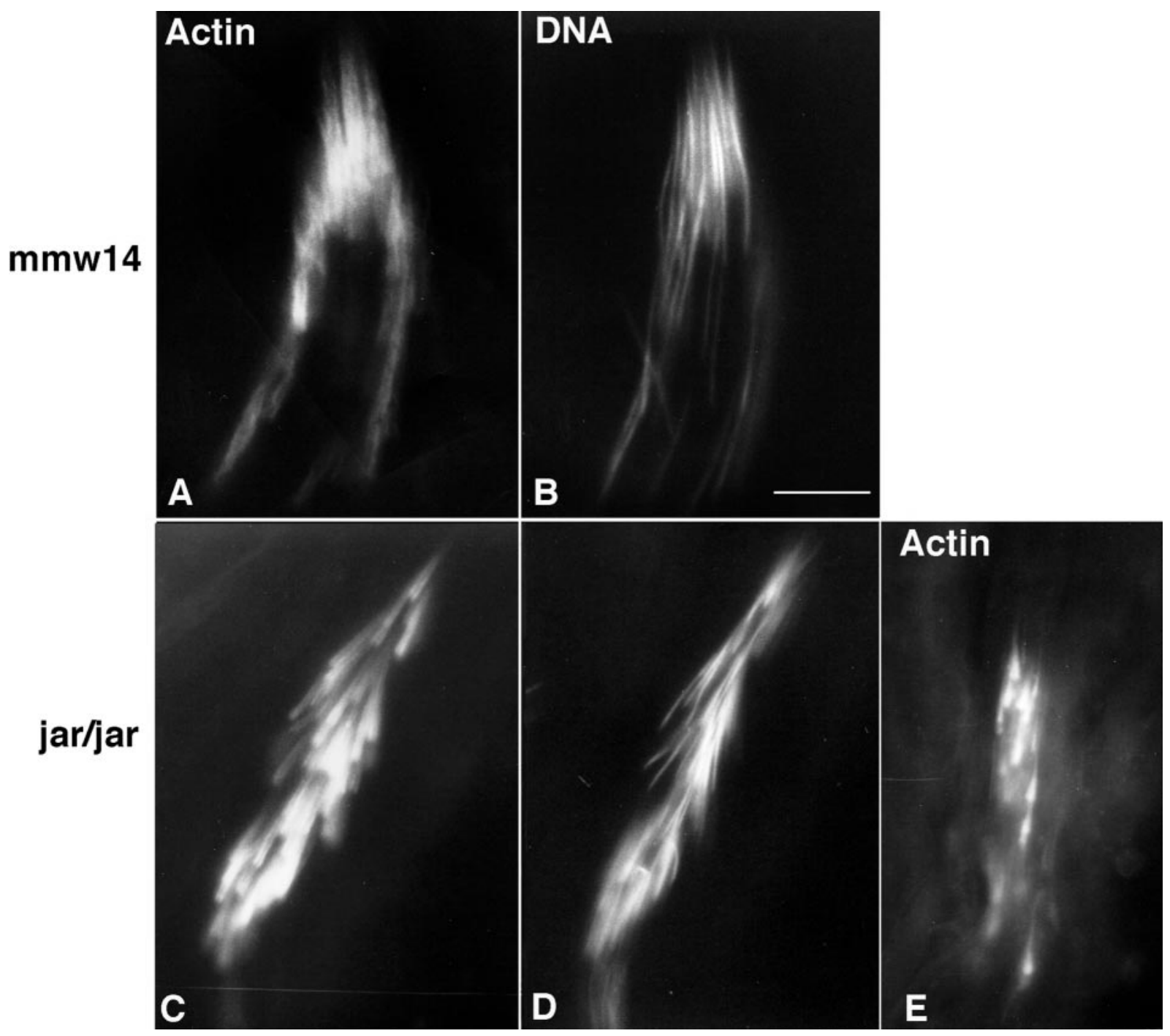

Figure 8. IC organization in 95F myosin mutants. In jarmmw14/Df(3R)S87-5 (labeled mmw14; A and B) and jar ${ }^{1} / j a r^{1}(\mathrm{C}-\mathrm{E})$ testes, the ICs are not aligned in register with one another, as seen in wild type. Testes were stained for either DNA (B and D) or actin (A, C, and E). The nuclei within a single cyst are positioned aberrantly, although actin is localized to each nucleus. 95F myosin is absent from the IC (our unpublished data). (E) Disrupted jar $^{1} /$ jar $^{1}$ IC that has progressed away from the nuclei. The nuclei and actin cones are strung out along the cyst, and the actin staining is diminished. Bar, $0.75 \mu \mathrm{m}$.

actin still forms investment cones, but the alignment of cones within each cyst is disrupted compared with wild type. It is not clear from our results whether this misalignment is a result of an initial defect in positioning of the nuclei or whether the complex begins to move in the absence of $95 \mathrm{~F}$ myosin, becoming disorganized as it progresses and dragging nuclei out of alignment. However, because spermatid elongation and nuclear condensation appear unaffected in mutant testes, it is unlikely that $95 \mathrm{~F}$ myosin plays a direct role in nuclear positioning.

After assembly of the complex, the IC progresses down the cyst, maintaining the alignment between investment cones of neighboring spermatids. 95F myosin becomes tightly associated with the base of the advancing investment cone, and this distribution is maintained as the IC progresses down the cyst to the waste bag. In the absence of $95 \mathrm{~F}$ myosin, either the IC never advances away from the nuclei, or its component cones advance out of register, resulting in a disrupted IC. This suggests that $95 \mathrm{~F}$ myosin is involved in IC movement. Based on the known functions for other myosins there are three possible models for the role of $95 \mathrm{~F}$ myosin during individualization: 1) a force-producing motor, which moves the IC; 2 ) an actin cross-linking structural protein; and 3) a motor involved in short-range movements, which are important for membrane reorganization.

The simplest model for the role of 95F myosin is that it provides the force that moves the IC along the length of the axoneme. If this is the case, when 95F myosin is absent the investment cones should not move away from their assembly site adjacent to the nuclei. In fact, we observe investment 


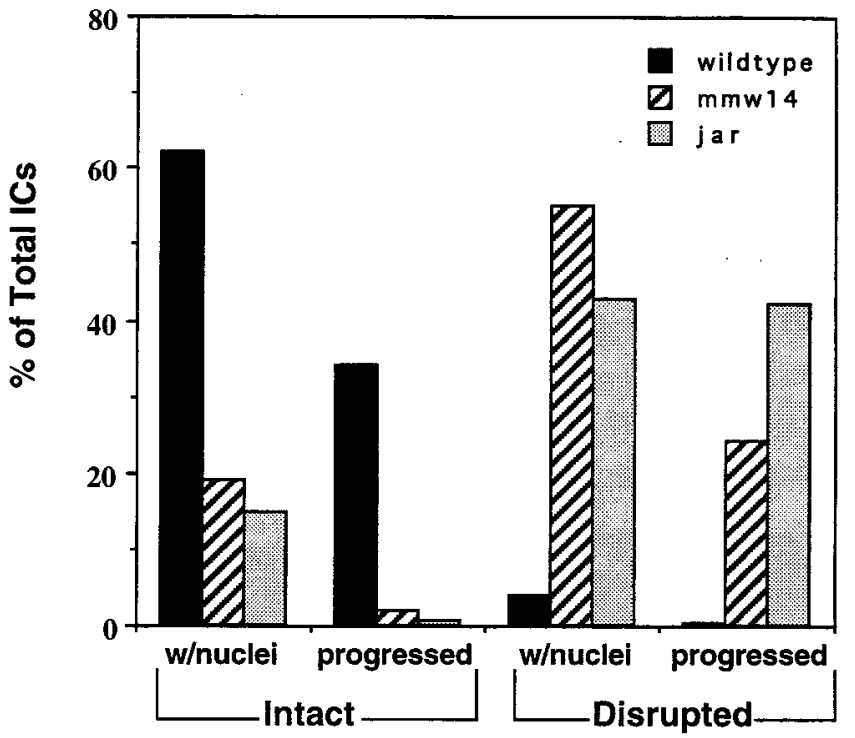

Figure 9. Quantitation of the effect of 95F myosin mutations on IC organization. Each IC was scored as either colocalizing with nuclei or progressed away from nuclei and then as intact or disrupted. The determination of whether an IC was intact or disrupted was by examining whether the individual investment cones were scattered (disrupted) or lined up in register with one another (intact). The majority of wild-type ICs are intact. In contrast, the majority of the mutant ICs are disrupted.

cones that have translocated away from the nucleus in the mutant, which make this model unlikely. In addition, in this model, 95F myosin would require some type of "track" on which to translocate down the spermatid. There is no obvious actin track preceding the IC. The microtubules that constitute the spermatid tail axoneme might form a track for movement, but it seems likely that a myosin would only be able to use these microtubules to move actin filaments and membrane in collaboration with microtubule-binding proteins and microtubule motors.

In other tissues, such as nervous system and posterior of the early embryo, 95F myosin colocalizes with D-CLIP-190, the Drosophila homologue of the vertebrate vesicle linker protein CLIP 170 (Lantz and Miller, 1998). Both CLIP-170 and D-CLIP-190 bind microtubules in vitro. To determine whether 95F myosin might interact with microtubules by way of D-CLIP-190, we examined the localization of DCLIP-190 in testes. D-CLIP-190 is not colocalized with 95F myosin in testes (our unpublished results). We hypothesize that if $95 \mathrm{~F}$ myosin produces force for movement during IC progression it does not do so by binding indirectly to microtubules by way of D-CLIP-190. This does not exclude the possibility that there is another as yet undiscovered CLIP family member or other microtubule-associated protein in Drosophila that could interact with 95F myosin in this process.

A second model for the role of 95F myosin is in stabilizing the actin structure as it moves. In this case, actin would assemble, and ICs would begin to move. However, because 95F myosin was absent, they would rapidly disassemble, leading to few progressed ICs. Our data show that in fact



Figure 10. Model for the role of 95F myosin during individualization. 95F myosin (myosin VI; purple) functions during individualization to recruit vesicles (green circles) to the actin-rich cortex underlying the spermatid plasma membrane. $95 \mathrm{~F}$ myosin transports vesicles a short distance along these actin filaments to the advancing edge of the investment cone. Once the vesicles arrive, they are incorporated into the remodeled membrane, which will enclose each sperm. The accumulation of $95 \mathrm{~F}$ myosin-rich vesicles at the site of incorporation leads to $95 \mathrm{~F}$ myosin enrichment in a ring at the base of the actin cone.

there is no substantial change in the proportion of ICs that have progressed. This model also does not easily explain why ICs and their associated nuclei are initially out of register in the mutant.

We favor the third hypothesis, that $95 \mathrm{~F}$ myosin might function to transport components important for individualization to the site of membrane growth. Progression of the investment cone down the spermatid bundle reorganizes the previously shared membrane so that each sperm is enclosed by a distinct membrane. This membrane remodeling is likely to involve an organized mechanism for delivery and incorporation of recycled and/or newly synthesized membrane and cytoskeletal components. We hypothesize that 95F myosin functions to recruit vesicles from the apical side of the IC (ahead of individualization machinery) to the site of membrane remodeling, the leading edge of the investment cone (Figure 10). 95F myosin would travel along cortical actin filaments toward the plasma membrane and release vesicles at the site of membrane remodeling. This model would explain the accumulation of 95F myosin in the band at the base of the leading edge of the investment cones. In this model, 95F myosin would not serve as the motor that generates force for IC movement down the spermatogenic cyst. Instead we postulate that the force that moves the IC is 
actin polymerization. This model is reminiscent of the mechanism of actin-based movement of Listeria monocytogenes (Theriot et al., 1992; Tilney et al., 1992).

If the membrane delivery and assembly machinery were defective, as we postulate is the case in the 95F myosin mutants, then the actin polymerization would eventually stall because of physical constraints on the membrane. As the ICs began to move, the nuclei and actin rings would be pulled out of alignment because of inefficient delivery of membrane or other membrane skeleton components to the site of force generation. This hypothesis would explain the observation that mutant testes contain a high number of disrupted, progressed ICs.

95F myosin might also (or instead) play a role in membrane endocytosis, which is likely to be required for this remodeling event. However, whether a role in endocytosis would cause $95 \mathrm{~F}$ myosin to accumulate at the base of the actin cones is unclear.

The data presented here do not allow us to eliminate 95F myosin as the force producer for IC movement or as a cross-linker of actin filaments. However, the idea that it plays a role in membrane transport is consistent with its function at other times in development and with the proposed association of myosin VI with Golgi vesicles and other membrane compartments (Buss et al., 1998; Bunn et al. 1999).

In the blastoderm embryo $95 \mathrm{~F}$ myosin is a particle transporter (Mermall et al., 1994; Mermall and Miller, 1995). This movement represents an actin-dependent translocation of vesicles or other cellular components into the newly forming membrane furrows. This type of rapid, directed incorporation of required components is quite similar to the reorganization that must occur upon individualization. In addition, $95 \mathrm{~F}$ myosin is expressed at the leading edge of follicle cells undergoing rapid shape changes as they migrate, which is also compatible with this interpretation (Deng et al., 1999).

Recently, Buss et al. (1998) have demonstrated that myosin VI localizes to the Golgi and the trans-Golgi network in mammalian cell lines. Myosin VI also is enriched at the leading edge of migrating human fibroblasts and is recruited to dynamic cell surface structures upon stimulation with epidermal growth factor. The authors suggest that myosin VI may be involved in exocytic membrane transport from the Golgi complex to the leading edge. In addition, the glucose transporter C-terminal binding protein (GLUT1CBP) has recently been shown to bind in vitro to myosin VI (Bunn et al., 1999). This interaction suggests a role for myosin VI as part of the glucose transporter complex important for specific membrane domain localization.

To date 15 classes of unconventional myosins have been recognized. However, little is known about their discrete functions in vivo (Cope et al., 1996). Mutations in myosins from several classes have revealed a wide range of phenotypes. In vertebrates, mutations in some unconventional myosins cause hearing defects, indicating that they play important roles in the hair cells of the neuroepithelium (for reviews, see Hasson, 1997; Steel and Brown, 1998). In fact, myosin VI is the gene affected in the Snell's waltzer mutant mouse. In these animals the cochlear and vestibular neurosensory epithelium degenerates soon after birth (Avraham et al., 1995). Within the hair cells of the inner ear, myosin VI localizes to the actin-rich cuticular plate, which anchors the stereocilia into the cytoplasm. Within this region, myosin VI associates with the pericuticular necklace, a structure rich in vesicles between the cuticular plate and the circumferential actin band (Hasson et al., 1997). It has been postulated that myosin VI may function to locally transport vesicles and organelles in this region. Although such a role would be consistent with our models of how 95F myosin functions in Drosophila syncytial blastoderm and spermatids, it has not yet been possible to correlate defects in hair cell vesicle trafficking with concomitant degeneration.

The partial loss-of-function mutations that we have characterized here provide important new information about class VI unconventional myosin function. Based on the excellent correlation between the subcellular localization of 95F myosin and the phenotype of the 95F myosin mutants, we have been able to develop a concrete model for membrane remodeling and cytoskeletal function that requires 95F myosin. Because the role of $95 \mathrm{~F}$ myosin appears similar in both spermatids and in syncytial blastoderm, further studies of this process promise to provide insight into unconventional myosins roles throughout development.

\section{ACKNOWLEDGMENTS}

We thank Dr. Steve Wasserman for discussions on the jaguar phenotype and sharing unpublished data, Dr. Chris Bazinet for suggestions on scoring of IC phenotypes and discussions on the $95 \mathrm{~F}$ myosin mutant defects, Dr. Cynthia Wagner for careful reading of the manuscript, and Dr. Julie Brill and Dr. Elizabeth Raff for helpful comments and testes fixation protocols. We also thank the Bloomington and Umea Drosophila stock centers for providing stocks. This work was supported by the Wellcome Trust (to M.B.), a National Research Service Award (to J.L.H.), and National Institutes of Health grant GM-43607 (to K.G.M.). W.-M.D. was supported by the Darwin Trust. A.D.R. was supported by a National Institutes of Health Cellular and Molecular Biology training grant.

\section{REFERENCES}

Avraham, K.B., Hasson, T., Steel, K.P., Kingsley, D.M., Russell, L.B., Mooseker, M., Copeland, N.G., and Jenkins, N.A. (1995). The mouse Snell's waltzer deafness gene encodes an unconventional myosin required for structural integrity of inner ear hair cells. Nat. Genet. 11, 369-375.

Bunn, R.C., Jensen, M.A., and Reed, B.C. (1999). Protein interactions with the glucose transporter binding protein GLUT1CBP that provide a link between GLUT1 and the cytoskeleton. Mol. Biol. Cell 10, $819-832$

Buss, F., Kendrick-Jones, J., Lionne, C., Knight, A.E., Cote, G.P., and Luzio, J.P. (1998). The localization of myosin VI at the Golgi complex and leading edge of fibroblasts and its phosphorylation and recruitment into membrane ruffles of A431 cells after growth factor stimulation. J. Cell Biol. 143, 1535-1545.

Castrillon, D.H., Gonczy, P., Alexander, S., Rawson, R., Eberhart, C.G., Viswanathan, S., DiNardo, S., and Wasserman, S.A. (1993). Toward a molecular genetic analysis of spermatogenesis in Drosophila melanogaster: characterization of male-sterile mutants generated by single P element mutagenesis. Genetics 135, 489-505.

Cope, M.J.T.V., Whisstock, J., Rayment, I., and Kendrick-Jones, J. (1996). Conservation within the myosin motor domain: implications for structure and function. Structure 4, 969-987.

Deng, W.-M., Leaper, K., and Bownes, M. (1999). A targeted gene silencing technique shows that Drosophila myosin VI is required for 
egg chamber and imaginal disc morphogenesis. J. Cell Sci. 112, 3677-3690.

Deng, W.-M., Zhao, D., Rothwell, K., and Bownes, M. (1997). Analysis of $P[$ Gal4] insertion lines of Drosophila melanogaster as a route to identifying genes important in the follicle cells during oogenesis. Mol. Hum. Reprod. 3, 853-862.

Fabrizio, J.J., Hime, G., Lemmon, S.K., and Bazinet, C. (1998). Genetic dissection of sperm individualization in Drosophila melanogaster. Development 125, 1833-1843.

Fuller, M.T. (1993). Spermatogenesis. In: The Development of Drosophila melanogaster, ed. M. Bate and A.M. Arias, pp. 71-147, Cold Spring Harbor, NY: Cold Spring Harbor Laboratory Press.

Hasson, T. (1997). Unconventional myosins, the basis for deafness in mouse and man. Am. J. Hum. Genet. 61, 801-805.

Hasson, T., Gillespie, P.G., Garcia, J.A., MacDonald, R.B., Zhao, Y., Yee, A.G., Mooseker, M.S., and Corey, D.P. (1997). Unconventional myosin in inner-ear sensory epithelia. J. Cell Biol. 137, 1287-1307.

Hime, G.R., Brill, J.A., and Fuller, M.T. (1996). Assembly of ring canals in the male germline from structural components of the contractile ring. J. Cell Sci. 109, 2779-2788.

Kellerman, K., and Miller, K.G. (1992). An unconventional myosin heavy chain gene from Drosophila melanogaster. J. Cell Biol. 119, 823-834.

Lantz, V.A., and Miller, K.G. (1998). A class VI unconventional myosin is associated with a homologue of a microtubule-binding protein, cytoplasmic linker protein-170, in neurons and at the posterior pole of Drosophila embryos. J. Cell Biol. 140, 897-910.

Lindsley, D., and Tokuyasu, K.T. (1980). Spermatogenesis. In: Genetics and Biology of Drosophila, 2nd ed., ed. M. Ashburner and T.R. Wright, pp. 225-294, New York: Academic Press.
Mermall, V., McNally, J.G., and Miller, K.G. (1994). Transport of cytoplasmic particles catalyzed by an unconventional myosin in living Drosophila embryos. Nature 369, 560-562.

Mermall, V., and Miller, K.G. (1995). The 95F unconventional myosin is required for proper organization of the Drosophila syncytial blastoderm. J. Cell Biol. 129, 1575-1588.

Mermall, V., Post, P.L., and Mooseker, M.S. (1998). Unconventional myosins in cell movement, membrane traffic, and signal transduction. Science $279,527-533$.

Rubin, G.M., and Spradling, A.C. (1982). Genetic transformation of Drosophila with transposable element vectors. Science 218, 348-353.

Stanley, H.P., Bowman, J.T., Romrell, L.J., Reed, S.C., and Wilkinson, R.F. (1972). Fine structure of normal spermatid differentiation in Drosophila melanogaster. J. Ultrastruct. Res. 41, 433-466.

Steel, K.P., and Brown, S.D. (1998). More deafness genes. Science 280, 1403.

Theriot, J.A., Mitchison, T.J., Tilney, L.G., and Portnoy, D.A. (1992). The rate of actin-based motility of intracellular Listeria monocytogenes equals the rate of actin polymerization. Nature 357, 257-260.

Tilney, L.G., DeRosier, D.J., and Tilney, M.S. (1992). How Listeria exploits host cell actin to form its own cytoskeleton. I. Formation of a tail and how that tail might be involved in movement. J. Cell Biol. $118,71-81$.

Titus, M.A. (1997). Unconventional myosins: new frontiers in actinbased motors. Trends Cell Biol. 7, 119-123.

Tokuyasu, K.T., Peacock, W.J., and Hardy, R.W. (1972). Dynamics of spermiogenesis in Drosophila melanogaster. I. Individualization process. Z. Zellforsch. 124, 479-506. 